\title{
Model-free adaptive control for the heading angle of a UAV
}

\author{
Di $\mathrm{LI}^{1, *}$, and Kun SHU ${ }^{1}$ \\ ${ }^{1}$ China Shipbuilding Industry corporation 723 , Yangzhou, China
}

\begin{abstract}
The difficulty of accurately modeling the aerodynamics of the heading angle of unmanned aerial vehicles is well known. Furthermore, it is difficult to control the heading angle of an unmanned aerial vehicle (UAV) with a model-based method under the influence of wind disturbances. To address this problem, a novel model-free control strategy is proposed. First, a self-organizing type-2 fuzzy brain emotional learning network model is used to estimate the PPD (pseudo partial derivative). Finally, the stability is guaranteed by designing the Lyapunov function. Meanwhile, the performance of the proposed method is further verified by high-fidelity semi-physical simulations.
\end{abstract}

\section{INTRODUCTION}

Unmanned aerial vehicles (UAVs) have been used in many applications, and there are a variety of control methods used in the control of UAVs, which include model-based and data-based methods. The model-based control scheme is prone to pollution by uncertainties and disturbances, and the mathematical model of the heading angle of UAVs is difficult to obtain. The data-driven control (DDC) method does not contain the mathematical model information of the controlled process within the design process explicitly or implicitly; it only uses online or offline process data to design the controller. Data-driven control technology is widely used in transportation [1], machinery manufacturing [2], chemical production [3] and motor control [4]. Currently, many kinds of DDC approaches have emerged, such as PID [5-6], model-free adaptive control (MFAC) [7-8], virtual reference feedback tuning [9-10], and iterative feedback tuning [11-12].

In this paper, a novel MFAC method is proposed for the heading angle control of UAVs. The candidate controller is DLC. However, the parameters are nonlinear and complex, so a fuzzy neural network is used to tune the parameters of the online DLC, which is piped into the proposed MFAC scheme and makes the system have more powerful capabilities for adapting to and tackling nonlinearity.

Some studies have proven that the control input data stream is chattered by a combination of uncertainties through different kinds of pathways. To solve this problem, some studies have proven that collaborating with artificial neural networks enhances the control performance under uncertainty. The concept of brain emotional learning (BEL) networks was defined by LeDoux in 1991[13-15]. Compared to other neural networks, a brain emotional learning network can achieve faster learning speed and better approximation capability due to its special structure; BEL networks have two parts: an orbitofrontal cortex and an amygdala. In recent years, BEL has been used for various fields, such as prediction, identification, and control[16-27]. However, these fuzzy logic sets introduced above are all based on type-1 networks. However, type 2 networks have more flexibility in handling uncertainties than type 1 networks. Therefore, authors have studied type- 2 fuzzy neural networks [28-30] and a type-2 fuzzy brain emotional learning network; additionally, a robust compensation was combined in [31].

Motivated by the above discussions, this paper proposes a model-free fuzzy-neuron adaptive control scheme to control UAVs. The main contributions of this paper include the following. A self-organizing type-2 fuzzy brain emotional learning network model is used to estimate the PPD. This model depends only on the input and output data and is proposed for the heading angle control of UAVs; this model is different from modelbased control algorithms that require exact dynamic model knowledge from the plant. The proposed model takes advantage of type-2 fuzzy theory and BELN, and there are no initial rules. The parameters are tuned simultaneously by the gradient approach. Numerical simulations have been performed to demonstrate the effectiveness of the proposed method. This paper is organized as follows: Section 2 introduces the dynamics of the heading angle of a UAV. Section 3 introduces the concept of interval type-2 fuzzy BELN. In Section 4, the overall control framework for the heading angle of UAVs, obtained by combining a self-organizing type-2 fuzzy brain emotional learning network and a model-free control method, is proposed; additionally, the stability of this proposed scheme is demonstrated. Section 5 shows the simulation results. Finally, the conclusions are given in Section 6. 


\section{The dynamic heading-angle of UAV}

The attitude of a UAV can be expressed as:

$$
\begin{aligned}
& \dot{\psi}=\omega \\
& J \dot{\omega}+c \omega+d=\tau(a, r)
\end{aligned}
$$

Where $\psi$ represents the yaw angle, $\omega$ represents the rate of angle $\psi, J$ represents the inertial, $c$ represents the Coriolis, $d$ represents the external disturbance, and $\tau(a, r)=[\tau(a), \tau(r)]$ represents the effects of rudder and aileron. Defining $\tau(a, r)=u(a, r), y=\omega$, then:

$$
J \dot{y}+c y+d=u_{a, r}
$$

The above equation can be transformed as follows by using the first-order Euler method:

$$
\begin{aligned}
& \frac{y(k+1)-y(k)}{\Delta T}=-\frac{c(k)}{J(k)} y(k)-\frac{d(k)}{J(k)}+\frac{u_{a, r}(k)}{J(k)} \\
& y(k+1)=M(k) y(k)-D(k)+N(k) u_{a, r}(k)
\end{aligned}
$$

The above equations can also be expressed as follows by employing the DLC technology defined by Hou and $\mathrm{Bu}$ (2011):

$$
\Delta y(k+1)=\Phi(k)\left[\Delta y(k), \Delta u_{a, r}(k)\right]^{T}
$$

The PPD in the above equation has the following expression:

$$
\Phi(k)=H_{y, u}(k-1)+\left[\frac{\partial y(k+1)}{\partial y(k)}, \frac{\partial y(k+1)}{\partial u_{a, r}(k)}\right]
$$

where a unique solution of $H_{y, u}(k-1)$ can be found for $\Delta y(k) \neq 0, \Delta u_{a, r}(k) \neq 0$

\section{The Interval Type-2 Fuzzy Brain Learning Network (IT2FBELN)}

Here, the nonlinear dynamics have evolved in the DLC, and we know that it is not easy to calculate these timevarying parameters. However, the PPD can actually be estimated with some algorithms, such as neural networks and neuron-fuzzy inference systems. This paper adopts the self-organizing IT2FBELN, which combines the advantages of both the brain emotional learning network and the type-2 fuzzy system, to estimate the PPD. The self-organizing IT2FBELN is designed as a six-layer architecture, as shown in Fig. 1, which shows the structure of IT2FBELN. The proposed IT2FBELN is constructed with six layers, which are listed below:

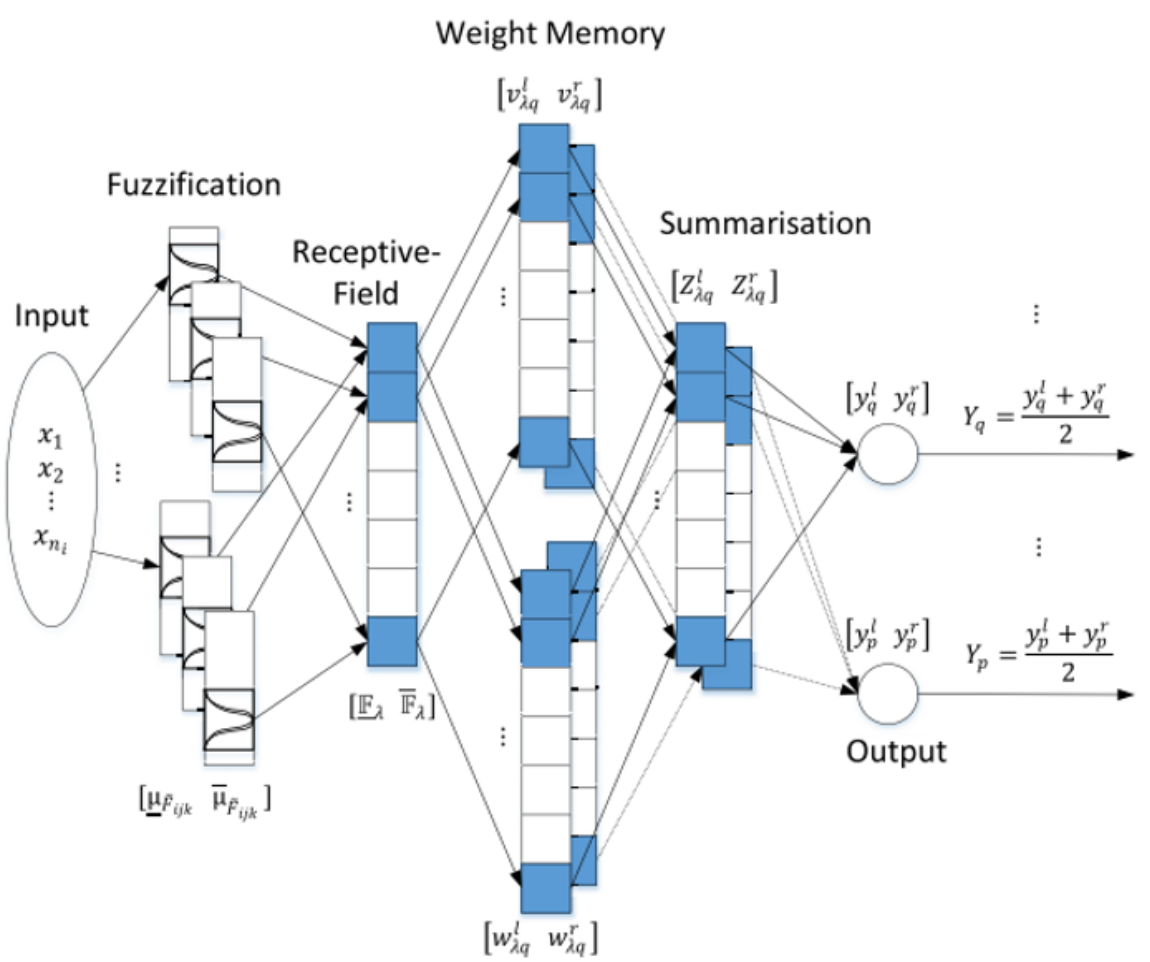

Fig. 1 Architecture of the IT2FBELN

1). The input space: The nodes are $X=\left[x_{1}, x_{2}, \cdots x_{n_{i}}\right]^{T}$, where ${ }^{n_{i}}$ represents the number of input signals, and all data from this layer will be transmitted to the next space without any computation.

2). The MF space: The Gaussian activation function is adopted to finish the fuzzification by using the interval type-2 membership function. The Gaussian membership function can be represented as:

$$
\begin{array}{r}
\mu_{i i j k}\left(x_{i}\right)=T\left(x_{i}, m_{i j k}, \sigma_{i j k}\right) \\
=\exp \left(-\frac{\left(x_{i}-m_{i j k}\right)^{2}}{2 \bullet \sigma_{i j k}^{2}}\right)
\end{array}
$$

where $\mu_{\tilde{F} i j k}\left(x_{i}\right), m_{i j k}, \sigma_{i j k}$ represent the membership, uncertain mean, and uncertain variance for the ith input, respectively, and $m_{i j k} \in\left[\underline{m}_{i j k}, \bar{m}_{i j k}\right]$; hence, lower 
membership degree $\underline{\mu}_{\tilde{F} i j k}$ and upper membership degree $\bar{\mu}_{\tilde{F} i j k}$ for each input of ${ }^{\mu_{\tilde{F} i j k}}$ can be defined as:

$$
\begin{aligned}
& \bar{\mu}_{F i j k}\left(x_{i}\right)=\left\{\begin{array}{l}
T\left(x_{i}, \bar{m}_{i j k}, \sigma_{i j k}\right), x_{i}<m_{i j k} \\
1, \\
T\left(x_{i j k}, m_{i j k}, \sigma_{i j k}\right), x_{i}>\bar{m}_{i j k} .
\end{array}\right\} \\
& \underline{\mu}_{\dot{F} i j k}\left(x_{i}\right)=\left\{\begin{array}{l}
T\left(x_{i}, \bar{m}_{i j k}, \sigma_{i j k}\right), x_{i}<\frac{m_{i j k}+\bar{m}_{i j k}}{2} \\
T\left(x_{i}, m_{i j k}, \sigma_{i j k}\right), x_{i}>\frac{m_{i j k}+\bar{m}_{i j k}}{2}
\end{array}\right\}
\end{aligned}
$$

3). Spatial firing layer: The rule is composed of both upper and lower MFs, and the firing strength is interval and can be calculated as follows:

$$
F_{\lambda}=\left[\underline{F}_{\lambda}, \bar{F}_{\lambda}\right]=\left[\prod_{i=1}^{n i} \underline{\mu}_{\hat{F} i j k}, \prod_{i=1}^{n i} \bar{\mu}_{\hat{F} i j k}\right]^{T}
$$

where $F_{\lambda}$ denotes the intervals of the firing strength of the MFs. The ni and $h$ represent the number of input signals and fuzzy rules, respectively.

4). Weight memory space: This layer contains two memory spaces, amygdala memory and orbitofrontal memory, which contain interval values because the firing space is interval. Therefore, the weight of the ith rule and kth output of the network are given as:

$$
\begin{aligned}
& \omega_{\lambda q}=\left[\omega_{\lambda q}^{l}, \omega_{\lambda q}^{r}\right] \\
& v_{\lambda q}=\left[v_{\lambda q}^{l}, v_{\lambda q}^{r}\right]
\end{aligned}
$$

5). Defuzzification space: The left- and right-most point values can be obtained:

$$
\begin{aligned}
& S_{n e t}=a_{q}-o_{q}=\sum_{\lambda=1}^{n \lambda} F_{\lambda q}\left(v_{\lambda q}-w_{\lambda q}\right)=\sum_{\lambda=1}^{n \lambda} F_{\lambda q} Z_{\lambda q} \\
& Z_{\lambda q}=\left[\begin{array}{ll}
Z_{\lambda q}^{l} & Z_{\lambda q}^{r}
\end{array}\right]=\left[\left(v_{\lambda q}^{l}-w_{\lambda q}^{l}\right)\left(v_{\lambda q}^{r}-w_{\lambda q}^{r}\right)\right]^{T} \\
& y_{q}^{l}=\frac{\sum_{\lambda=1}^{R} \bar{F}_{\lambda} Z_{\lambda q}^{l}+\sum_{\lambda=R+1}^{n \lambda} \underline{F}_{\lambda} Z_{\lambda q}^{l}}{\sum_{\lambda=1}^{R} \bar{F}_{\lambda}+\sum_{\lambda=R+1}^{n \lambda} \underline{F}_{\lambda}} \\
& y_{q}^{r}=\frac{\sum_{\lambda=1}^{R} \bar{F}_{\lambda} Z_{\lambda q}^{r}+\sum_{\lambda=R+1}^{n \lambda} \underline{F}_{\lambda} Z_{\lambda q}^{r}}{\sum_{\lambda=1}^{R} \bar{F}_{\lambda}+\sum_{\lambda=R+1}^{n \lambda} \underline{F}_{\lambda}}
\end{aligned}
$$

where $a_{q}=\sum_{\lambda=1}^{n \lambda} f_{\lambda q} v_{\lambda q}$ is the output of the amygdalalike memory and $o_{q}=\sum_{\lambda=1}^{n \lambda} f_{\lambda q} w_{\lambda q}$ is the output of the confrontational memory.

6). Output space: The output of this layer is an interval value, so average operations are used to obtain network outputs:

$$
y_{q}=\frac{y_{q}^{r}+y_{q}^{l}}{2}
$$

The output of IT2FCM cannot be directly transformed to a crisp set by a defuzzifier. The aim of type-reduction is to compute the center of a type 2 fuzzy set. At present, the iterative Karnik-Mendel (KM) algorithm and the enhanced Karenina-Mendel (EKM) algorithm are used to compute the center of an interval type-2 fuzzy set efficiently; the improved EKM is used here. The IT2FCM contains seven tunable parameters, and the updating rules of these seven parameters using the gradient descent method can be derived as:

$$
\begin{aligned}
& \underline{\hat{\hat{m}}}_{i \lambda}=\eta^{m} \cdot \underline{F}_{i \lambda}^{m} \cdot \frac{Z_{\lambda}^{l}+Z_{\lambda}^{r}}{2} \cdot s(\underline{e}(t)) \\
& \dot{\hat{\hat{m}}}_{i \lambda}=0 \\
& \dot{\hat{\sigma}}_{i \lambda}^{l}=\eta^{\sigma} \cdot \underline{F}_{i \lambda}^{\sigma} \cdot \hat{Z}_{\lambda}^{l} \cdot s(e(t)) \\
& \dot{\hat{\sigma}}_{i \lambda}^{r}=\eta^{\sigma} \cdot \underline{F}_{i \lambda}^{\sigma} \cdot \hat{Z}_{\lambda}^{r} \cdot s(e(t)) \\
& F_{q}^{l}= \frac{\underline{F}_{\lambda}}{\sum_{\lambda=1}^{L} \bar{F}_{\lambda}+\sum_{\lambda=L+1}^{n \lambda} \underline{F}_{\lambda}} \\
& F_{q}^{r}= \frac{\underline{F}_{\lambda}}{\sum_{\lambda=1}^{R} \bar{F}_{\lambda}+\sum_{\lambda=R+1}^{n R} \underline{F}_{\lambda}} \\
& \dot{\omega}_{\lambda q}^{l}=\beta\left[F_{\lambda}^{l} \cdot\left(u-d_{q}\right)\right] \\
& \dot{\omega}_{\lambda q}^{r}=\beta\left[F_{\lambda}^{r} \cdot\left(u-d_{q}\right)\right] \\
& \dot{v}_{\lambda q}^{l}=\alpha\left[F_{\lambda}^{l} \cdot\left(\max \left[0, d_{q}-a_{q}\right]\right)\right] \\
& v \cdot{ }_{\lambda q}^{r}=\alpha\left[F{ }_{\lambda}^{r} \cdot\left(\max \left[0, d_{q}-a_{q}\right]\right)\right]
\end{aligned}
$$

\section{The model-free IT2FBELN control for the heading angle of a UAV}

Theorem 1: Define the error of the heading angle as $\ddot{\psi}(k)=\psi_{d}(k)-\psi(k)$, the error of the angle rate as $\breve{\varpi}(k)=\varpi_{d}(k+1)-\varpi(k)$, and the estimation error of the the PPD as $\bar{\Phi}(k)=\hat{\Phi}(k)-\Phi(k)$. Then, the proposed controller for this system is given as:

$$
u(a, r)(k)=u(a, r)(k-1)+\frac{\eta(k) \hat{\Phi}(k)}{\lambda(k)+\|\hat{\Phi}(k)\|^{2}}\left[k_{1} \tilde{\psi}(k)+\tilde{\varpi}(k)\right]
$$

where $0<k 1<2,0<\lambda(k)<1,0<\eta(k)<1$, and then $\lim _{k \rightarrow \infty}\left[\psi_{d}(k)-\psi(k)\right]=0$ can be guaranteed through the above control law.

The discrete Lyapunov function can be defined as:

$$
V(k)=\frac{1}{2} \tilde{\psi}^{2}(k)+\frac{1}{2} \tilde{\varpi}^{2}(k)+\frac{1}{2} \tilde{\Phi}^{2}(k)
$$

Then:

$\Delta V(k)=V(k+1)-V(k)$

$\leq \frac{1}{2}\left[(1-k)^{2}-1\right] \ddot{\psi}^{2}(k)+\frac{\Gamma^{2}-1}{2}\left\{\left[\ddot{\varpi}(k)+\frac{\Gamma^{2}}{\Gamma^{2}-1} \Delta \varpi *(k+1)\right]^{2}\right\}$

From the above equation, we can see that $\Gamma^{2}-1<0$. That is, $\Delta V(k) \leq 0$. When $\tilde{\psi}(k)=0$ and $\check{\varpi}(k)=-\left(\Gamma^{2}\right) /\left(\Gamma^{2}-1\right)\left[\Delta \varpi^{*}(k+1)\right]$, then $\Delta V(k)=0$. 


\section{Simulations}

There is no need to obtain the physical dynamic model; however, the I/O data of the plant need to be collected to implement the proposed algorithm, which is compared with the PID controller and model-free adaptive controller (MFAC) in an autopilot simulation. The heading angle of the UAV under the fixed value for the angle rate and turbulent wind is shown in Fig. 2, where the angle rate is $0.1 \mathrm{rad} / \mathrm{s}$, turbulent wind is 5 knots, cruise velocity is $14 \mathrm{~m} / \mathrm{s}$, and altitude of the UAV is 100 $\mathrm{m}$.

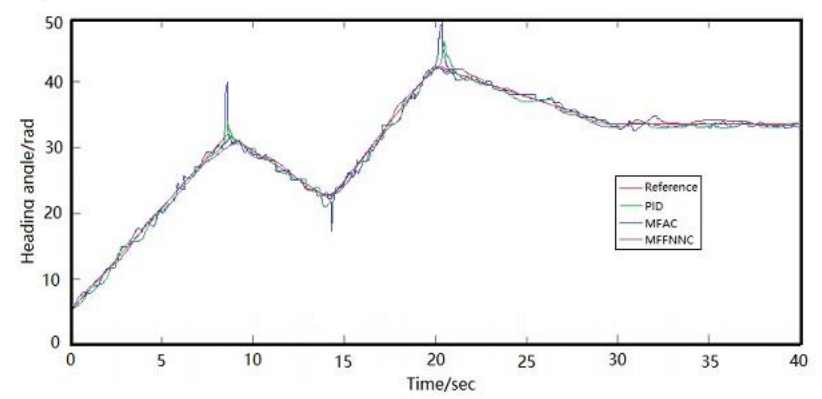

Fig. 2 Heading angle of the UAV under a fixed value for the angle rate and turbulent wind

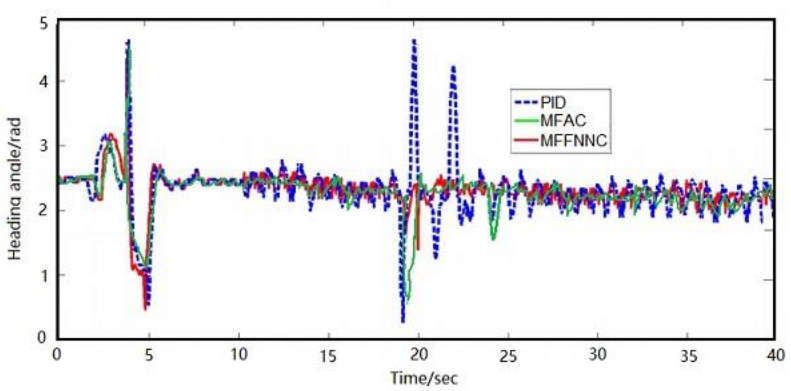

Fig. 3 Control inputs of aileron under a fixed value for the angle rate and turbulent wind

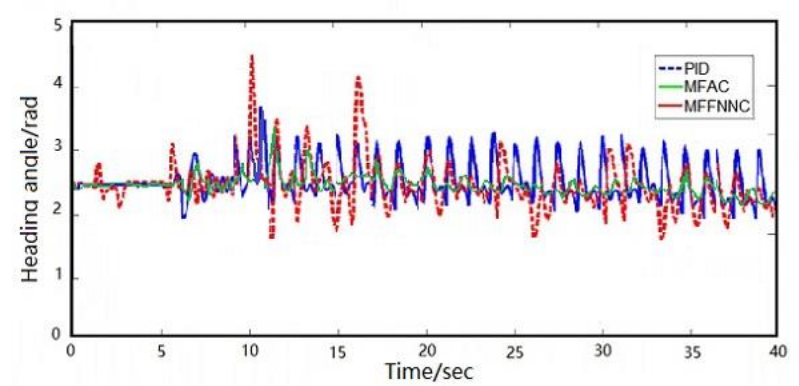

Fig. 4 Control inputs of the rudder under a fixed value for the angle rate and turbulent wind

From Fig. 2, we can see that the tracking error of the proposed control method is less than that of the PID controller and model-free adaptive controller [32], which indicates that the proposed method has better performance under turbulent wind conditions. Additionally, the control inputs are shown in Figs. 3 and 4, which demonstrate that the heading angle can be tracked well when using $\mathrm{I} / \mathrm{O}$ data and the proper controller.
From the simulation results, we can see that the performances of the proposed method, the PID and the model-free adaptive controller are similar but differ greatly when the heading of the vehicle is perpendicular to the direction of the wind (18-25 $\mathrm{s}$ in the figure). Additionally, the control inputs are presented in Figs. 8 and 9 .

\section{Conclusions}

A model-free fuzzy adaptive control strategy united with an interval type 2 fuzzy brain emotional learning network to control the heading angle of a UAV has been proposed in this paper. First, the nonlinear equation for the heading angle has been converted with dynamic linear technology, and the PPD is estimated by an interval type 2 fuzzy brain emotional learning network. The stability of the proposed control scheme is guaranteed. Finally, the performance of the proposed method is verified by high-fidelity semi-physical simulations.

\section{References}

1. S. Formentin, P.D. Filippi, M. Corno, et al. Datadriven design of braking control systems. IEEE Transactions on Control Systems Technology, 2013, 21(1): $186-193$.

2. G. Wang, Z. Huang. Data-driven fault-tolerant control design for wind turbines with robust residual generator. Control Theory and Applications Iet, 2015, 9(7): 1173-1179.

3. D. Xu, B. Jiang, P. Shi. A novel model-free adaptive control design for multivariable industrial processes. IEEE Transactions on Industrial Electronics, 2014, 61(11): 6391-6398.

4. R. M. Cao, H. X. Zhou, Z. S. Hou. Data-driven model-free adaptive precision control for linear servo system. Control Theory and Applications, 2012, 29(3): 310-316.

5. K. J. Astrom, T. Hagglund, and A. Wallenborg, Automatic Tuning of PID Controllers. Research Triangle Park, NC, USA: Instrum. Soc. Amer., 1988.

6. J. Sivag, A. Datta, and S. P. Bhattacharyya, "New results on the synthesis of PID controllers," IEEE Trans. Autom. Control, vol. 47, no. 2, pp. 241-252, Feb. 2002.

7. Z. S. Hou, The Parameter Identifification, Adaptive Control and Model Free Learning Adaptive Control for Nonlinear Systems. Boston, MA, USA: Northeastern Univ., 1994.

8. Z. S. Hou and W. H. Huang, "The model-free learning adaptive control of a class of SISO nonlinear systems," in Proc. IEEE Amer. Control Conf., Jun. 1997, pp. 343-344.

9. G. O. Guardabassi and S. M. Savaresi, "Virtual reference direct design method: An off-line approach to data-based control system design," 
IEEE Trans. Autom. Control, vol. 45, no. 5, pp. 954-959, May 2000.

10. M. C Campi, A. Lecchini, and S. M. Savaresi, "Virtual reference feedback tuning: A direct method for the design of feedback controllers," Automatica, vol. 38, no. 8, pp. 1337-1346, 2002.

11. H. Hjalmarsson, S. Gunnarsson, and M. Gevers, "A convergent iterative restricted complexity control design scheme," in Proc. 33rd IEEE Conf. Decision Control, Dec. 1994, pp. 1735-1740.

12. H. Hjalmarsson, "From experiment design to closed-loop control," Automatica, vol. 41, no. 3, pp. 393-438, 2005.

13. J. LeDoux, Emotion and the limbic system concept, Concepts Neurosci. 2(1991) 169-199.

14. J.E. LeDoux, Emotion circuits in the brain, Annu. Rev. Neurosci. 23 (2000) 155-184.

15. C.-M. Lin, C.-C. Chung, Fuzzy brain emotional learning control system design for nonlinear systems, Int. J. Fuzzy Syst. 17 (2) (2015) 119-128.

16. J. Ayubi, A. Omidi, S.M. Barakati, P. Ayubi, Short term load forecasting based on brain emotional predictor, in: Proc. EPDC, 2015, pp. 37-41.

17. M. Jafari, R. Fehr, L.R.G. Carrillo, H. Xu, Brain emotional learning-based intelligent tracking control for unmanned aircraft systems with uncertain system dynamics and disturbance, in: Proc. ICUAS, 2017, pp. 1470-1475.

18. M. Jafari, S.B. Shuraki, Speed control of a digital servo system using brain emotional learning based intelligent controller, in: Proc. PEDSTC, 2013, pp. 311-314.

19. S. Khorashadizadeh, M. Mahdian, Voltage tracking control of DC-DC boost converter using brain emotional learning, in: Proc. ICCIA, 2016, pp. 268272.

20. E. Lotfi, Mathematical modeling of emotional brain for classification problems, in: Proc. IAM, 2013, pp. 60-71.

21. E. Lotfi, M.-R. Akbarzadeh-T., Adaptive brain emotional decayed learning for online prediction of geomagnetic activity indices, Neurocomputing 126(2014) 188-196

22. M.A. Sharbafi, C. Lucas, R. Daneshvar, Motion control of omni-directional three-wheel robots by brain-emotional-learning-based intelligent controller, IEEE Trans. Syst. Man Cybern. C 40 (6) (2010) 630-638

23. C.-F. Hsu, C.-T. Su, T.-T. Lee, Chaos synchronization using brain-emotionallearningbased fuzzy control, in: Proc. SCIS, 2016, pp. 811816.

24. H.-S. Milad, U. Farooq, M.E. El-Hawary, M.U. Asad, Neo-fuzzy integrated adaptive decayed brain emotional learning network for online time series prediction, IEEE Access 5 (2017) 1037-1049
25. Chun-Fei Hsu, Tsu-Tian Lee.Emotional Fuzzy Sliding-Mode Control for Unknown Nonlinear Systems. International Journal of Fuzzy Systems, 19(3):942-953, 2017

26. Wubing Fang, Fei Chao, Chih-Min Lin, Longzhi Yang, Changjing Shang, andChangle Zhou,An Improved Fuzzy Brain Emotional Learning Model Network Controller for Humanoid Robots, Frontiers in Neurorobotics, 2019,1-16

27. Jing Zhao, Chih-Min Lin3, and Fei Chao.Wavelet Fuzzy Brain Emotional Learning Control System Design for MIMO Uncertain Nonlinear Systems, Frontiers in Neurorobotics, 2019,1-11

28. X.J. Chen, D.Li, X.B.Yang, and H.M.Li. Radar Emitter Signals Identification with a Optimal Recurrent Type 2 Wavelet Fuzzy Neural Network, International Journal of Aeronautical Space Science, DOI 10.1007/s42405-018-0055-X.

29. X.J. Chen, D.Li, X.B.Yang, and Y.C.Yu, Optimal Type II Fuzzy Neural Network Controller for Eightrotor MAV. International Journal of Control, Automation and Systems 15(4) :1960-1968, 2017.

30. X.J. Chen, D.Li, X.B.Yang, and Y.C.Yu, Identification recurrent type 2 fuzzy wavelet neural network and L2-gain adaptive variable sliding mode robust control of electro-hydraulic servo system(EHSS), Asian Journal of Control, 20(5):111,2018

31. Chao, Fei, Zhou, Dajun, Lin, Chih-Min, Yang, Longzhi, Zhou, Changle and Shang, Changjing. Type-2 Fuzzy hybrid controller network for robotic systems, IEEE Transactions on Cybernetics, 1-15, 2019

32. S.L. Zhao, X.K.Wang, D.B.Zhang, and L.C. Shen, Model-Free Fuzzy Adaptive Control of the Heading Angle of Fixed-Wing Unmanned Aerial Vehicles, Journal of Aerospace Engineering, 30(4):1-10, 2018 